\title{
Central Body Rotation Drives Orbital Revolutions
}

\section{Paul Schroeder}

Wind Lake, WI, USA

Email: Pshrodr8@aol.com

How to cite this paper: Schroeder, P. (2018). Central Body Rotation Drives Orbital Revolutions. Journal of Geoscience and Environment Protection, 6, 70-84. https://doi.org/10.4236/gep.2018.612005

Received: May 17, 2018

Accepted: December 10, 2018

Published: December 13, 2018

Copyright $\odot 2018$ by author and Scientific Research Publishing Inc. This work is licensed under the Creative Commons Attribution International License (CC BY 4.0).

http://creativecommons.org/licenses/by/4.0/

\begin{abstract}
Prior to the development of physics as a science it was sufficient to have motion that offsets a perpendicular gravitational force and continues forever. The total absence of any friction could be assigned to the "void" of space. But perpetual motion and empty space are now seen as fallacies. Continuous motion needs a driving force for impetus.
\end{abstract}

\section{Keywords}

Physics, Motion, Gravitational Force

\section{Introduction}

I have developed a replacement gravity theory over the years called External Gravitation.

I offer here a reason that Kepler's third law worked for the first 6 planets. An elementary activity in the universe is motion of celestial bodies relative to each other. An all inclusive physical gravity theory should focus upon spatial motions.

A function of physics is to want to understand fields and matter better by searching for their components in the atomic universe. Particle theory is the search within a three dimensional whole for one dimensional pieces such as molecules, atoms, protons, electrons, photons, etc. Our perspective view of a whole field can also be broken into linear pieces much like physicists break it into particles. The newer physics has been string theory which is the search for two dimensional linear connections or flows. My model introduces Paeps as one dimensional particles which serve as individual waves when in motion. That motion is the linear two dimensional piece called a beam. My External Gravity model implies joining particle theory and string theory with emphasis on longer strings, i.e. spatial beams moving throughout space. 
Assumptions of External Gravity:

1) The universe is infinite and isotropic.

2) Actions are the motions of matter. There is no perpetual motion of matter without a perpetual source causing the motion. Gravity is the perpetual source.

\section{Fundamentals of External Gravitation}

A successful gravity system must address and improve many of the confusing issues of physics and astronomy, finally leading to new areas of investigation. By resolving my gravity concepts with relevant physics issues I have developed different views about numerous concepts including how rotating centers cause orbiting as measured by Kepler's third law of planetary motions. Fundamental concepts include:

1) Gravity is a push rather than attraction. As such it solves "action at a distance”.

2) Gravitation is best pictured as lines rather than fields. Lines help analyze and contemplate a linear push. Pressure gradients that summarize the situation inhibit analysis.

3) Gravitation functions as beams pushing from all directions upon every point of space.

Thus matter takes on spherical shapes. Equilibrium is the balance of vertical pushes.

4) The push implies a velocity of the beams. Motion of beams is confirmed by waves within the beams.

5) Gravitation pushes as if it contained moving particles-Paeps-particles applying external pressure.

6) Gravity particles can't be matter. They would cause too much heat upon impact.

7) Radiation particles strike matter with impact. The amount of impact depends on the wave frequency and altitude.

8) Radiation rarely or slightly penetrates masses due to its wave structure. Longer wave gravity radiation penetrates and extends its push throughout. Thus Paep gravity beams replicate long wave radiation.

9) Paep beams, like radiation waves move at velocity C. Gravity carries radiation.

10) Gravity beams are the structure of space.

11) The universe is infinite and consists of moving Paep gravity beams throughout. Gravity is the aether others refer to. Likewise gravity is the indetectible background.

12) Gravity beams mostly penetrate matter. Atoms are mostly empty space. Paep waves interacting with atomic particles modify each other.

13) Penetrating gravity beams exit the mass and are modified. Beams may be diminished, modified into heat and light radiation, and/or have its motion redirected. 
14) Impacted matter particles are modified into radiation or different matter. The sun's eternal power is caused by continuous penetration and exiting of gravity beams.

15) Paeps can be redirected by spin of atomic particles or by the spin of the whole mass. Density is the spin of mass relative to local equilibrium.

16) Redirected Paep beams exit in a bent path. They curve throughout their travels.

17) The gravitational push at a mass surface "nets out" diminished exiting beams with undiminished incoming beams resulting in what we have called attraction gravity.

18) Undiminished gravity beams flow in all directions across the mass surface, not just downward. A small extra flow is the counterclockwise flow of exiting beams bent by the rotation of the mass. The flow matches the mass's rotation yielding local equilibrium.

19) Newton said orbital motion continues absent external forces. The implied void of space can't exist given radiation, meteors, and solar winds. A motive force is needed.

20) Newton's "motive" external force was centrifugal force, a force with no source.

Newton's inertia is more properly defined as; "adhering to the local flow of gravity".

21) Rotating bodies spin counterclockwise relative to their central body. Central body bent gravity beams add to atmospheric "revolution". The sum of bent gravity beams from earth's rotation and from the sun causes winds on earth.

22) Solar gravity beams are the solar wind when passing by earth. Magnetosphere pictures are attempts to represent bent gravity beams. Between planet and sun bent beams from each will collide, creating a small void.

23) Orbitals incur lateral pushes of gravity beams from both the planet and the sun.

The rotation push by spinning bodies upon their orbitals decreases with altitude.

24) Orbital revolution rates must be less than their central body rotation rate. Rotation of the master (sun) adds to the local central body (planet) push upon moons.

25) Sufficient bending of radiation beams and interaction with other beams creates mass. Electrons are beam crossings.

26) Magnetism is the net of gravity beams when beams from one direction are redirected.

27) The nuclear-strong force is simply the sum of gravitation pushing from all directions.

28) Charge is simply the direction of flow. Anti-gravity is pushing in the opposite direction.

29) From Paep gravity thru EM radiation extending to mass defines the spectrum of existence, all tied to the wave structure pictured for radiation. 
30) Three dimensional waves are best pictured as coils.

31) Diminished gravitation occurring locally within the sun or stars is replenished by the gravitational stretching of light beams into microwaves, then radio waves, and further into Paep gravity beams as they travel from very distant stars.

\section{Transmissions}

Light/radiation and external pushing gravity are the two transmissions which convey the nature of celestial bodies. Einstein pointed out an issue with light where significant motion of bodies relative to light signals distorts their location and time measures. Light signals likewise can be influenced by motion of the observer, which action is specified as aberration. When beams approach an observer they may arrive off line, bent inward, due to the motion of the observer. While the linear motion of sources is insignificant the transmission of that light signal is subject to the revolution motion of that celestial source body. A conundrum is that light conveys the picture of motion while the motion influences the transmission of the light beam.

We shall first discuss aberration here primarily to introduce a few concepts and begin to understand why planets revolve.

\section{Orbital Aberration, Sun to Earth}

One important topic to be addressed in preparation is the structure of space. We will look at orbiting first with respect to aberration of effects between earth and the sun. After that we will diagram and analyze the relative measure of orbital velocities. There are gravity theories based upon vortices throughout space. If a vortex is analyzed there needs be interacting pressures from multiple directions. To consider such things in detail we need to orient our thinking toward a linear view of causes and avoid generalizing actions into pressure gradients. Herein we will introduce some concepts of a pushing gravity, which serves as an inversion of attraction gravity, in order to realize that "there is no aberration of this gravity".

In order to understand the aberration for light etc. one inspects the relative motions of the bodies involved in the transmission of the EM waves. Reviewing all motions of relevant bodies is especially important for transmissions within the solar system such as sun to earth transmission.

There are four relative motions of bodies, three of which are important for aberration. The fourth, which is the sun's displacement caused by earth's gravity, is negligible. The first motion of interest is the motion of earth around the sun. Analyzing this we first imagine two circles and a straight line connecting the two circles and serving as a beam of light. The paper we draw on is static space and ignores the revolution motion. Given the beam takes 8 minutes to transmit, and earth moves to the left, counterclockwise, during that time, the beam will pass behind earth. To correct for this we select a beam aimed ahead on earth's path which we intend will hit exactly the center of earth in 8 minutes. That straight 
line beam will appear to bend slightly backward to earthly observers upon arrival pitting earth's motion of $29.8 \mathrm{KM} / \mathrm{sec}$ against the outward velocity $\mathrm{C}$ of the beam. The bending is called aberration. This diagram is useful for light coming from beyond an orbit, but creates an incorrect picture of sun to earth transmissions.

The next, and most important motion, is the rotation of the sun. Consider an observer on the sun and the launch of the light beam. Say a beam is launched straight up like an arrow toward a chosen point. After 8 minutes is the beam still straight up from the solar observer? Since the sun has rotated a bit (its period of rotation is 24 days), it is above and somewhat behind the observer and the point of origin. The beam has not gone straight up! What does straight up mean? Perhaps the motion should be represented by a curve drawn to compensate for the rotation of the solar surface. Then the arrow can be pictured as straight up at some future times. In fact this representation does occur in some form as the launch site was moving sideways while the beam headed upward. The first assumption about the form of the sideways motion is that the beam will move toward the left at the sun's rotation rate, which is $2 \mathrm{~km} / \mathrm{sec}$ pitted against the upward velocity of C. But this offset, at $2 \mathrm{~km} / \mathrm{sec}$ vs $29.8 \mathrm{~km} / \mathrm{sec}$. of earth's revolution velocity, is insufficient to explain earth's revolution; it bends the beam only $1 / 15^{\text {th }}$ as much as needed. Note that we are considering only the lateral effect of the perpendicular push in a static space. The other offset perspective is that of angular velocity. This curving initial offset is a much more significant factor. The sun rotates in 24 days, which is 15 times faster than earth's revolution. Applying this rotation to an EM transmission throughout would have the beam arriving at earth from behind. This would be a large reverse aberration.

Essentially there is gradually fading lateral equilibrium at the surface of both earth and the sun. Space is not a static medium. The angular velocity is the initial offset to $\mathrm{C}$ at the surface of the sun. But the angular velocity applied to radiation beams diminishes as the beam departs the source. To understand source to receiver (sun to earth) transmissions vs the motions of earth we must consider the real condition of space.

External gravity is long wave radiation beams traveling at velocity $\mathrm{C}$. These beams are the fabric of space similar to the nature some assign to an aether. Gravity beams behave as does other EM radiation. Gravity beams also can penetrate masses. It is those beams exiting from the sun that simulate the previous analysis for light. Ultimately the gravity beams push the planet in its motion. The exiting beams acquire the rotation of the sun. If we assumed the solar rotation angularly pushed these beams throughout they might push earth 15 times as fast its actual revolution rate. The sun rotates in $1 / 15^{\text {th }}$ of our year. To account for the difference, recognize that the density of the original beams and of space itself diminish with distance. Think of master beams as continually modifying themselves by:

1) The absorption of beams angling inward, and

2) some of the beam parts normally angle away slightly. 
The solar atmosphere which rotates with the sun's surface gradually loses its connection with increasing altitude and its rotation contribution slows to that of the surface. That happens because the sum of the master beam representing our gravity beam absorbs increasing portions of beams that did not originate at the sun but angled in. In any case, there is no aberration in gravity as it is the motive force of the planet's motion. The whole explanation to Kepler's third law comes from netting together the beams that have gained bending by exiting from the sun.

Since external gravity and light are both EM radiation beams, the absence of gravity aberration applies similarly to light. But gravity is also the cause of the whirling space in which light beams follow the flow and arrive without aberration.

The following definitions of aberration give a general summary of why there is not aberration in the common sense between central bodies and their orbitals.

Definition of aberration (1)

1) A deviation from the proper or expected course. See Synonyms at deviation.

2) The apparent displacement of the position of a celestial body in the direction of motion of an observer on Earth, caused by the motion of Earth and the finite velocity of light.

a) doesn't suggest a straight line which I show is observer dependent and thus is not a real or the proper course.

b) assumes a straight line offset by earth's motion. I show why that is overcome by solar rotation.

\section{Pushing Gravity Theories}

Previous pushing gravity theories suffer from concern about particles inhibiting the flow of orbitals. One recent modification of pushing gravity suggests an ultra high speed of pushing gravity particles. This was probably not part of leSage's original pushing gravity theory, but comes from LaPlace, Van Flandern, and others as incorrect solutions to pushing gravity theories, all of which ignore curvature. The solution using bending/curvature is entirely new here.

\section{Newton}

Our ideas of space develop from Newton's system of celestial mechanics. Essentially Newton applied gravity to Kepler's third law and realized that the mass of each body would then be a factor. Heavier orbitals would be pulled down more intensely. Newton added mass to the equation, thus providing for the continuation of the orbital motions. That is a solution for orbiting if all factors remain unchanged, but it ignores the cause of original and continuation of the motion. Under my assumptions motions of matter lacking impetus are not permanent. This is not a theory predicting Kepler's law. The effort here is to add an impetus to Newton's system of the world. Newton's orbital control infers that orbiting occurs because nothing interferes with the linear forward component of motion. 
So, to him, the linear component of motion is perpetual, devoid of outside influences such as friction. By extension the rotation of bodies themselves, like the orbital motion of revolution, lack outside influence and would also be perpetual. We now understand that space is not a void.

Our physical science, which depends on cause and effect, must come forward and correct these ideas. The cornerstone of attractive forces and of frictionless space comes from application of Newton's laws even though he refuted "attraction" gravity and action without contact.

A discussion of gravitational force by Newton follows:

"For here I design only to give a mathematical notion of those forces, without considering their physical causes. -Wherefore the reader is not to imagine that by those words, I say where take upon me to define the kind, or the manner of any action, the causes or the physical reason thereof, or that I attribute forces, in a true and physical sense, to certain centers (which are only mathematical points); when at any time I happen to speak of centers as attracting, or as endued with attractive powers". "You sometimes speak of gravity as essential and inherent to matter. Pray do not ascribe that notion to me; for the cause of gravity is what I do not pretend to know.” (Newman, 2003).

In addition, Newton had said "he would not refute gravity as a motive particle is it didn't hinder the motion of orbitals."

Building a theory on an absence of influence by anything was necessary to build a world system and an absolute space theory. But in today's world of technical detail knowledge, the absence of a source needs to be discarded in favor of an ongoing impetus.

Newton's views competed with and overcame the whirlpool theories of Descartes as a source of orbital motion. The rotation velocities of Descartes' whirlpool representation of motions of space itself couldn't equal velocities in central spin experiments such as rotating fluids in a bucket nor if rotating within a fluid medium that extends to infinity. In neither example do the velocities or actions of the fluids simulate velocities as calculated using the formula of Kepler's third law. These examples probably led to disinterest in Descarte's model. What is needed and provided herein is a logical source of rotations leading to a different measure of central controlled whirling motions.

\section{Orbiting}

Our goal is to explain orbiting by explaining how a body rotating can cause motion for a second body. To get there we will suggest approaches, find the shortcomings, make corrections, and then try again.

Consider questions from inspecting Figure 1. Imagine first a 2 dimensional picture of 3 equatorial circles A, B and C. Circle A is larger. It spins counterclockwise and has lines radiating out from it. Assume the lines are attached to and rotate with the circle. The key question is what will the lines do when encountering matter in their path? They may 


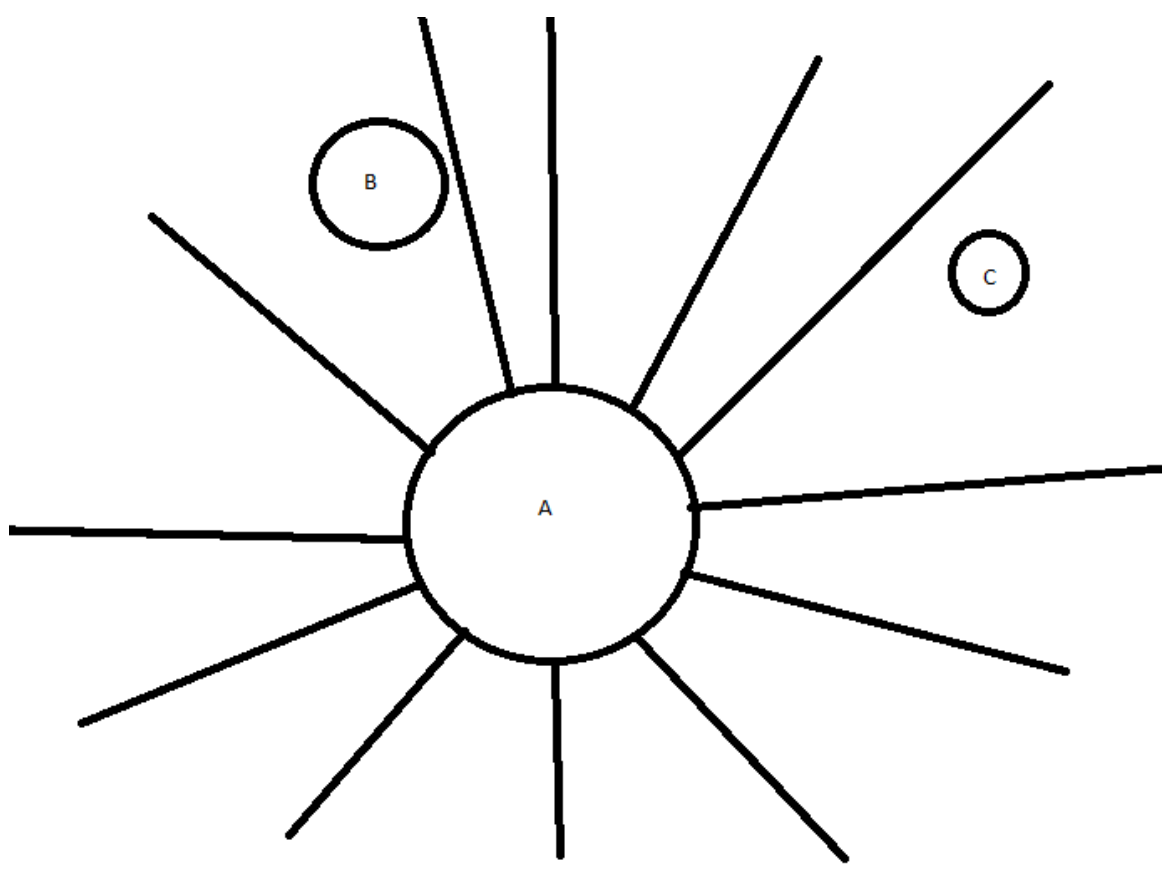

Call A the sun, B the earth, and C Mars.

Figure 1. Radiation exiting the sun.

1) not interact with the matter, they may

2) partially push on the matter, or they may

3) push and carry the matter along with them.

If they don't interact with matter we have no orbiting so we will concentrate on the other 2 options.

Assume the lines to be massive so that they can push upon and carry with them anything they encounter in their path-(option 3). A line encounters and pushes circle B to the left, somewhat like the force we call centrifugal. This push will also cause motion by B angularly away from A as B rolls out further along the line. There is no retaining wall.

We can't have the circle moving away from A in our analogy. Orbital motion essentially retains a body's distance from the central body as it moves around. Our example produces a linear motion, and we need an attraction toward center as a partial offset. We need something to attract/push B toward A with exactly the right force to balance the leftward motion caused by the central body spin. This attraction is a centripetal force and we call it the attraction of gravitation. Let's view gravity by reversing the direction of the lines so that they are now pushing radiation beams. These lines come from remote space and arrive at our circles. Imagine lines impacting circle B similar to those shown for circle A. No direction is primary for these lines so they arrive equally from all directions. Let's call them pushing lines that create a force. Coming from all directions they balance each other out so the net force is zero unless some modification occurs unbalancing the net effect. We get the imbalance by defining a limit on the pressure of the lines from one direction. We do that by specifying there must be 
less lines coming from A than from elsewhere. So A must diminish or block the push of the lines which exit from its surface. There becomes a "net" attraction of B by $\mathrm{A}$.

We have developed the beginnings of an external gravity system. Netting the sideways pushes with the attraction pushes in concert results in orbiting. But in the example given, the left pushing radiation beams will push any object around the center in the same time frame. If a second body such as circle $C$ is located further than $B$ is from $A$, it will travel faster but its rotational velocity will be the same. That assumes the pushing beams likewise dominate motion at all distances, that the original radiation lines retain the same leftward carry ability at all distances.

At this point we have orbital angular motion for B which corresponds with the surface spin of A. But this is not how orbiting works as we know that spatial objects do not retain their position in space over the same surface point permanently. In this example the period of revolution would be independent of the distance $\mathrm{R}$. There does happen to be an example to this unusual relationship as the earth nearly retains its position over a point on the moon's surface. This can only occur due to the moon being a minimal source of earth's motions.

What we are seeking is a relationship between orbital motions that varies with depending on the distance from a central body-sun. This relationship has been quantified in a complex formula for the closest 6 planets by Kepler's third law.

Kepler's formula can be simplified to $\mathrm{KT}=\mathrm{R}^{3 / 2}$ for each planet, where

$\mathrm{T}$ is the period of a full orbit cycle,

$\mathrm{R}$ is the distance of the center of the planet (B) from the surface of the sun,

(for elliptical orbits, $\mathrm{R}$ is the major axis.)

$\mathrm{K}$ is constant for every planet connecting their periods to a central body action.

We called the lines coming out of A radiation beams. As such they may logically be unable to push sideways if we assume they are like light which moves rapidly outward at the speed of $\mathrm{C}$. Viewed from the side of its path a light beam has no mass. But we do know light creates some pressure upon impact. Light has waves that emulate a particle upon impact. Light is only considered massless if at rest. For our radiating lines to push, they must have some motion toward the mass being pressured. Radiation traveling at speed $\mathrm{C}$ is usually considered linear motion. If the waves bend a bit sideways a miniscule amount of their push can be in the lateral direction. One might call the sideways impact glancing blows. So any bending of the radiation line toward the impacted mass should provide motion to the mass.

We give this bending some attention here with Figure 2. The sun A on the left emits beams toward earth $B$ on the right. Bending of the beam relative to circle $B$ must occur given our definition that $\mathrm{A}$ is spinning relative to $\mathrm{B}$. For a radiation line to move up against B it has traveled to our left. The bend up in Figure 2 serves as the leftward flow. That leftward motion L occurs while the beam moves 


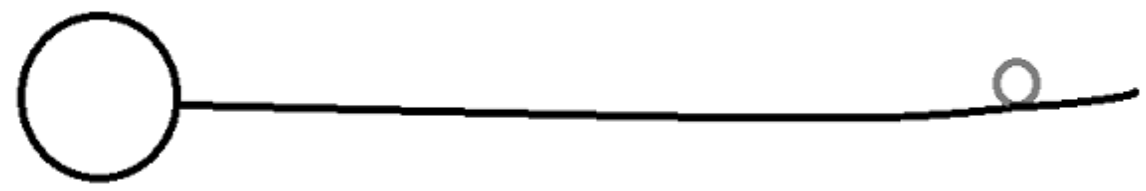

Picturing a gravity beam curving by the time it arrives at earth

Figure 2. Bent transmission beams.

outward at speed C. So $\mathrm{L} / \mathrm{C}$ is the amount of sideways push delivered to B. This is a very small portion of the speed of light.

The tangential push of radiation can't instantly cause the known motion of planets. It would take a long time to accelerate a static body in space to achieve a constant velocity. Nothing in this system could propel something like a manmade space ship into orbit. We can however imagine the natural gravity system's tangential push being sufficient to retain existing motion of an orbital already in motion. A tangential push is simply not strong enough to accelerate a body to its velocity $\mathrm{L}$. But once achieved, the motion can be perpetual the pushing is permanent.

We noted above radiating gravity beams exiting circle A carry and provide less pressure upon B than do uninhibited beams. This suggests that radiation arriving from multiple directions can carry differing forces or pressures depending on events along their paths. The forces vary especially for beams that had penetrated and exited a mass or for beams that had been bent by some action. These events modify the net pressure. Radiation lines/beams come from all directions toward circle B. Now if we move B further from A We note that the percentage of beams striking $B$ which came from directly A decreases. Therefore the number of beams that are diminished by $A$ and impact $B$ is a lesser percentage of all beams striking $B$ at any one time so that the gravitational attraction of $B$ by $A$ is less. Also the number of beams that are bent by $A$ and impact $B$ is a lesser percentage of all beams striking $B$ at any one time so that leftward push of B by A is less.

The tangential push now depends on the distance from center in some form. The beams departing the central mass are only a portion of the beams striking all points on planet B. The portion diminishes as B is drawn further from A. The decrease is proportional to the amount of the circumference of the circle around $\mathrm{B}$ that $\mathrm{A}$ occupies. Thus the pressure that the beams from A to B impart decreases as the radius $\mathrm{R}$ increases since those beams occupy a diminishing portion of the circle of influence acting upon B.

As an orbital revolves in its circle, it retains its distance from the center and continues to receive the same percentage of its beams from the center, ie. from the sun. Those are the bent beams that can push sideways. We can now conclude that the revolution period for orbitals is dependant on the radius $\mathrm{R}$ from the central body's surface. But we are missing something. If $\mathrm{R}$ were the factor com- 
mon to the periods of planets then the velocities would all be the same and the period would be a simple function of the circumference.

The analysis here is limited to circular orbits for simplicity. One can extend the logical thought to elliptical orbits.

Our example has related equatorial circles and been performed in two dimensional space. Next we must consider that there is altitude and thus a third dimension involved in these bodies since they are globes rather than circles. The area of the surface of a globe is a function of $R^{2}$ rather than just $R$. The measure for transferring an effect from the surface to some distant higher altitude point is to relate the sphere at that altitude to the sphere of the globe. That means the effect is diminished by $\mathrm{R}^{2}$. Brightness is one effect that is assumed to diminish by $\mathrm{R}^{2}$ and is used to determine relative distance of stars. Newton's formula for centripetal gravitational attraction between bodies also diminishes by $\mathrm{R}^{2}$. For these to be correct, the effect itself must be circular on the surface and distributed equally around the globe. We assume that is true for both attraction gravity and stellar brightness.

Do gravity pushes bent by the sun diminish in the $\mathrm{R}^{2}$ form? Applying $\mathrm{R}$ squared would have the more distant planet losing speed faster and thus revolving even slower than it does relative to an inner planet. For Kepler's formula $\mathrm{KT}=\mathrm{X} ; \mathrm{R}^{2}$ doesn't work as $\mathrm{X}$ nor does $\mathrm{R}$. Kepler determined that $\mathrm{KT}=\mathrm{R}^{3 / 2}$. We wish to determine the geometry this comes from. We do notice that a tangential push is not a circular radiating effect as is brightness. The tangential push can't be the same at all altitudes of the globe as it decreases from the equator to the poles. So the bent gravity push is unlike direct outward radiation and fails rules for using $\mathrm{R}$ squared. In fact the tangential push reduces to zero above the poles. That reveals why orbitals rarely orbit spinning central bodies above higher latitudes.

The missing $\mathrm{R}^{1 / 2}$ component is composed of the rotation provided by all lines bisecting the sphere and exiting at latitudes other than 0 , latitudes up to \pm 90 degrees. Consider that one of those lines exits at $45^{\circ} \mathrm{N}$ latitude. This steeply angled beam would not directly affect any but very nearby large globes. Beams more nearly parallel to the plane, and which therefore may contact orbitals depending on their inclination, are the ones we have yet to consider. The total rotation effect applied to space is the net of these lines which range in effect from $\mathrm{R}$ to zero as the latitudes increase.

The general cause of revolutions dictated by the $3 / 2$ power is as follows: Space is defined by $\mathrm{X}, \mathrm{Y}, \mathrm{Z}$ components-three dimensional. To affect all space by one event equally is represented as a cubic power. To affect all space by a diminishing radial event one uses a squared power. To affect a linear part of space takes a first power. And finally we can affect all space by an effect diminishing horizontally and then diminishing vertically. We arrive at a type of midpoint which in power geometry is a sq root. The mid point of affecting all space is the third dimension divided by the second power. 
We try here to break the general rules into specific geometry. Beyond the net push sourced from the equator, it is necessary to consider the additional rotational push provided to orbitals by the rotation of the central body's non-zero latitude beams of gravity. Prior to Newton's contribution of mass, Kepler's formula assumed orbitals to be geometric points. All non-equitorial penetrating beams intersect the equatorial plane somewhere. Some intersect it within the sun and some beyond the confines of the sun. We simply note here that that the intensity of the rotation circle emitted at non equatorial regions of the sun provide weaker orbital drive to orbital than do equatorial beams.

Picture 1 showed the equatorial circles on an $\mathrm{x} y$ plane. We viewed them from above which is positive on the $\mathrm{z}$ axis. This is an ideal perspective since we can view almost all rotations throughout the universe as being counterclockwise from this view. Now Figure 3 shows two circles on the $\mathrm{x} z$ plane which we view from the side on the y plane. The equator is drawn such that it bisects the sun here. We see beams that miss and fail to impact the planet. All beams that cross the equatorial plane inside the sun, outside the sun but prior to the planet, or beyond the planet do not provide rotational push. That leaves significantly few of the third dimensional beams that contribute. We can plot them by switching our reference point to the orbiting planet. We draw a X Z circle around the planet which includes the sun. Then the pertinent beams yield a vertical line containing those arriving from the north pole of the sun to the south pole of the sun. Switching reference point has related the two circles by $\mathrm{R}^{1 / 2}$.

We review by drawing the mentioned $3^{\text {rd }}$ dimensional contributions. Figure 3 shows a vertical view of the sun as seen from the ecliptic. From this new picture we can see beams pass through the sun at different angles. Those of interest now enter the sun at one latitude and exit at a latitude that is closer to the equator. Beams passing through the sun toward a planet which are not flat to the ecliptic will also diminish as did our equatorial beams if these beams strike the planet. However another factor that diminishes total impact of these beams is whether they make contact with the planet or not. The upper line misses the planet entirely by passing over its North Pole. Had the planet been nearer to the sun the line would have impacted the planet. The lower line shows that beams may initially miss the planet, subsequently participate in the contact and would miss again if the distance $\mathrm{R}$ were greater. Again for beams angling toward the planet,

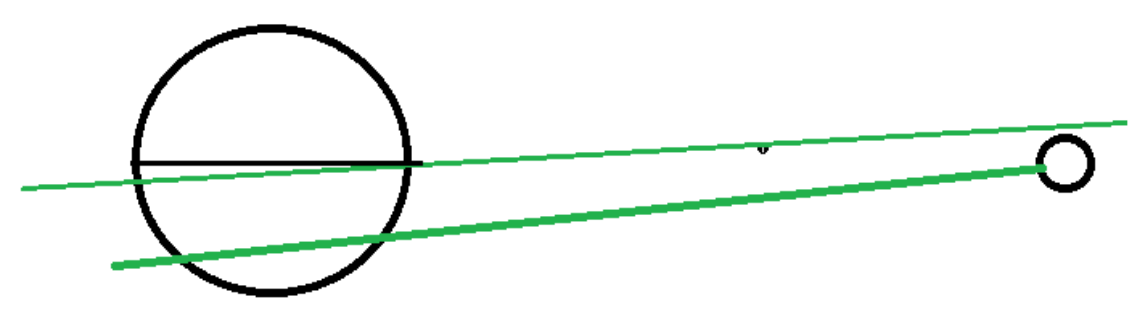

Figure 3. Gravity beams from the left enter the sun and exit in paths that may or may not impact a planet. 
the sum of all their contacts will decrease with distance. For each line draw a right triangle with side one the radial line to the planet, side two the perpendicular line altitude of the solar latitude exit point, and the hypotenuse the beam to the planet. Variations in the radial distances will determine how many of the lines impact the planet. Each line is a factor of the sq root of its particular altitude. So the sq root of the altitudes, plus and minus, determine the tangential push effects of beams inclined to the ecliptic plane.

The factor that summarizes the impacts of these higher latitude lines is a measure of sq root of the suns vertical diameter. Then the sum of factors affecting an orbitals velocity, and thus its period of revolution is $\mathrm{R}$ (for equatorial lines) $\times 2 \mathrm{R}^{1 / 2}$ (for non-equitorial lines) and thus $\mathrm{kR}^{3 / 2}$. Ultimately the spatial flows calculated by these two factors merge together to provide the overall whirling of space.

(An odd side view is that Kepler's constant $\mathrm{K}$ has the value of 5 for all planets. The only constant used for relating $R$ to the circle of $T$ would be pi. Since we have a circle and a sq root of same, perhaps the constant connects to pi + sq root pi. That is $3.14=1.77$ or 4.91 which somewhat approximates 5 .)

Consider the converse of the solar rotation causing orbital revolutions. In Newton';s construction and in my analysis the actions of the planets participate as additioinal causes. The net gravity at planet surfaces affects the sun. As we know, Newton's theory recognizes the planetary pull on the sun makes gravitation interact between two bodies with each applying force upon the other. For discussing planetary effects on the sun, the planet serves as the frame of analysis. The planets want to push the sun in orbit but by the time it has moved at all the planet has moved and its push direction changed significantly. There is orbiting by the sun but it is contained well within the sun. This shows the reverse interaction by revolutions with rotations where revolution causes rotation. The net result is that each planet causes a bit of rotation in the sun as their net gravity pushes on it. The sum of the push from all planets causes the solar 24 day rotation rate.

\section{The Starting Altitude Reveals How Central Bodies Controls Revolutions}

Though central body rotation causes orbiting, the smallest participating rotation is not the surface. In reality one would expect a direct connection between rotation rate of source with revolution rate of its orbitals. The revolution rate of the central body's surface cannot participate in these calculations as its radial distance from itself is 0 . Actually the surface cannot participate due to a counterclockwise rotation within its atmosphere. The apparent motion of the orbital would br a clockwise rotation of the orbital when considered by a point on the central body surface. Surface rotations are always faster than orbital revolutions.

Kepler's formula works as well for moons of gas planets such as for Juptier or Neptune. Secondary orbitals such as moons incur lateral pushes of gravity beams 
from the planet. But there are also enhancing lateral pressures from the sun that must be eliminated. To determine the system starting point the solar lateral push contribution must be eliminated before the formula can work.

To understand this, consider winds on earth. Winds are partly caused by bent gravity beams from the sun's rotation. This effect adds to the local earth's body's push of rotation. Winds flow toward the East, counter to earth's rotation. They must be overcome which occurs at high altitudes where the earth rotation is not overcall by atmosphere flow. Moons can only exist beyond the lower altitudes where solar bent gravity beams offsets planets rotation. Wind and jet streams exceed the rotation of earth up to an altitude (somewhat beyond the geosynchronous altitude) at which point the atmosphere finally begins to rotate counterclockwise along with the earth rotation. Above that the atmosphere/aether will revolve slower than the earth's surface does. Beyond their synchronous point, the tangential push by spinning suns or planets upon their orbitals decreases with orbital radius in conjunction with Kepler's law. That synchronous region serves as sort of the base distance from which the central body rotation can match and satisfy the formula of planetary periods of revolution. Remember that orbital revolution rates must always less than their central body rotation rate. Earth has no set of orbitals that can determine exactly where above the synchronous point Earth's rotation participates.

The sun also has a rotating atmosphere. So the starting point for planetary orbits begins near the helio-synchronous point. Solar atmospheric rotation results from the bent gravity beams from the planets trying to push the sun resulting in its rotation. That rotation will be greatest before it reaches the sun from each planet. Thus maximum solar region rotation is in the suns atmosphere. The resulting synchronous point may be near 11 solar diameters above the sun's surface. Mercury and the other planets that follow the Bode law revolve beyond that radial distance.

\section{Conclusion}

What I have presented here is intended to match existing knowledge but invert the source concept and introduce the concept of pushing. To remain viable the system reacts similarly as attraction does to existing math formulae. Any potential modifications relate to the proportion of $\mathrm{C}$ that is offset by the small speed of central body rotations throughout space.

Besides that, do note that attraction gravity is a linear action. As such many concepts such as strong force and antigravity have appeared to describe what that gravity cannot describe. Pushing gravity covers action from all directions and limits the need for newer concepts. There are recent experiments with findings that conflict with attraction gravity but are compatible with pushing gravity.

I don't believe there are any recent models suggesting the reason for $\mathrm{R}^{3 / 2}$ in Kepler's third law. However Kepler himself was nearing this explanation with his prehensive force. The logic became apparent when the rotation of the sun be- 
came known and Kepler used it. Apparently afterward the logic became buried in Newton's attraction gravity, inertia, and his calculus. Throughout the years many scientists and cosmologists have promoted pushing gravity models and all ignored the revolution of the sun.

A further interaction of revolutions with rotations is seen for interactions with equal sized bodies. Two such bodies drive each other by means of their escaping bent gravity beams. They revolve around a virtual central point. Essentially the orbits become $1 / 2$ the size of solar system orbits. Then the period squared becomes $1 / 4$ as much.

There are numerous physical relationships which are reanalyzed using the concept of External Gravitation (Schroeder, 2010, 2011a, 2011b, 2016a, 2016b, 2016c).

\section{Conflicts of Interest}

The author declares no conflicts of interest regarding the publication of this paper.

\section{References}

Newman, J. (2003). Isaac Newton, Principia; in James Newman. The World of Mathematics, $1,261$.

Schroeder, P. (2010). Gravity from the Ground Up. Proceedings of the NPA, 7, 498-503.

Schroeder, P. (2011a). Get 'Real' about Gravity. Proceedings of the NPA, 8, 521-529.

Schroeder, P. (2011b). Ignoring Newton's Hints Brought Scientific Chaos. Proceedings of the NPA, 8, 521-529.

Schroeder, P. (2016a). Infinity and Reality (The Universe Is Otherwise Part 2a). In D. De Hilster, R. De Hilster, \& N. Percival (Eds.), Proceedings of the CNPS (2016) (pp. 178-182). The John Chappell Natural Philosophy Society.

Schroeder, P. (2016b). Structure and Gravity (The Universe Is Otherwise Part 2b). In D. De Hilster, R. De Hilster, \& N. Percival (Eds.), Proceedings of the CNPS (2016) (pp. 183-192). The John Chappell Natural Philosophy Society.

Schroeder, P. (2016c). Infinity and Pushing Gravity Lead to Revelations (The Universe Is Otherwise Part 2c). In D. De Hilster, R. De Hilster, \& N. Percival (Eds.), Proceedings of the CNPS (2016) (pp. 193-199). The John Chappell Natural Philosophy Society. 